\title{
Las demasiadas traducciones. Sobre la Oficina para el Visado de Traducciones y el Cuerpo de Inspectores Técnicos de Traducción $(1942-1946)$
}

\author{
Octavio Moreno Cabrera ${ }^{1}$
}

Recibido: 8 de octubre 2019/ Aceptado: 15 de mayo 2020

Resumen. La década de los 40 fue el escenario de un intenso debate sobre el exceso de traducciones que, a juicio de las instituciones del Régimen franquista, poblaban el mercado editorial, poniendo en riesgo "el porvenir de la cultura española" y "la gloria imperial de nuestra bella lengua". Por esta razón, en 1942 se creó, en el seno de la Sección de Política Cultural del Instituto Nacional del Libro Español, una Oficina para el Visado de Traducciones en la que colaboraban activamente los miembros del recién creado Cuerpo de Inspectores Técnicos de Traducción. De esta manera, la publicación de versiones españolas de obras extranjeras no solo requería la aprobación previa de su contenido y valor moral por parte de la Sección de Censura de Libros de la Delegación Nacional de Propaganda, sino también la valoración positiva de su calidad y pureza lingüísticas por parte de la Oficina para el Visado de Traducciones. En el presente trabajo, se propone una aproximación a ese nuevo mecanismo que pretendía erradicar toda influencia extranjera sobre el idioma español.

Palabras clave: Franquismo, INLE, libro, censura, traducción.

\section{[en] Too many translations. About the Office for the Validation of Transla- tions and the Board of Technical Translation Inspectors (1942-1946)}

\begin{abstract}
The 1940s was the scene of an intense debate about the excessive number of translations that, in the opinion of the institutions of the Franco regime, populated the publishing market in Spain, thus putting at risk «the future of Spanish culture» and «the imperial glory of our beautiful language». Accordingly, an Office for the Validation of Translations was created in 1942 within the Cultural Policy Section of the National Institute of Spanish Books, in which members of the newly created Board of Technical Translation Inspectors actively collaborated. In this way, the publication of the translation of foreign works into Spanish not only required the prior approval of the Book Censorship Section of the National Propaganda Delegation with regard to content and moral value. It also needed a positive assessment of the translation's linguistic quality and purity by the Office for the Validation of Translations. The present work proposes an approach to this new programme that aimed to eradicate all foreign influence on the Spanish language.
\end{abstract} Key Words: Franco regime, INLE, book, censorship, translation.

Sumario. 1. Un nuevo mecanismo de control. 2. Confusión entre censura y visado. 3. Creación de la Oficina para el Visado de Traducciones. 4. Traducciones inspeccionadas y traducciones exentas. 5. Tareas desarrolladas por la Oficina para el Visado. 6. Tasas y sanciones. 7. Los inspectores. 8. Las quejas de los editores. 9. Fin del visado y disolución del cuerpo de inspectores. 10. Conclusiones.

Universidad Complutense de Madrid

ocmoreno@ucm.es 
Cómo citar: Moreno Cabrera, O. (2020) Las demasiadas traducciones. Sobre la Oficina para el Visado de Traducciones y el Cuerpo de Inspectores Técnicos de Traducción (1942-1946), en Estudios de Traducción $10,213-229$.

\title{
1. Un nuevo mecanismo de control
}

\begin{abstract}
La Vicesecretaría de Educación Popular [...] mantiene [su] política de fomento cultural mediante la censura de publicaciones, la intervención de planes editoriales y el visado de las traducciones, que ha impedido la publicación de ciertas versiones que eran deshonor de la lengua castellana. Tal censura aviva y no mata. Porque no mata sino a lo que ya merecía muerte por sí mismo ( $A B C$ 1944).
\end{abstract}

Durante los primeros años de la dictadura liderada por el general Francisco Franco, la política del libro se caracterizó por un fuerte intervencionismo. El Régimen quería hacer de él "soporte de exaltación de todos los valores patrios" y "arma de combate en pro de la moral y de la educación de[1] pueblo" (INLE 1942a: 15). Con este objetivo, se creó, en 1939, el Instituto Nacional del Libro Español (INLE). A partir de ese momento, los editores vieron complicarse los trámites burocráticos previos a la autorización de publicación y distribución de sus libros. Aún más, si cabe, en el caso de querer verter una obra literaria al castellano.

Como el resto de títulos, las traducciones debían ser remitidas a la Sección de Censura de Libros de la Delegación Nacional de Propaganda dependiente de la Vicesecretaría de Educación Popular para que, tras la oportuna revisión, se expidiese, en su caso, la tarjeta de color azul que autorizaba a imprimir el libro. El censor revisaba atentamente la obra para tachar cualquier elemento que pudiera ir en contra del Nuevo Estado. El visto bueno del censor permitía además la inclusión de la obra en los planes editoriales y, en el caso concreto de las traducciones, la obtención de las divisas necesarias para el pago de los derechos de autor al extranjero (INLE 1942h). Pero solo si la traducción quedaba justificada a partir de la "impecable ortodoxia" y "debido mérito literario" de la obra original (INLE 1943c).

La década de los 40 fue el escenario de un intenso debate sobre el exceso de traducciones (Moret 2002: 64-68, Andrés 2012: 47-57) y, sobre todo, "malas traducciones" que, a juicio de las instituciones del Régimen, poblaban el mercado editorial (Herrero 1942: 5-6), poniendo en riesgo "el porvenir de la cultura española" (INLE 1943c) y "la gloria imperial de nuestra bella lengua" (INLE 1943a). Por esta razón, en 1942 se creó, en el seno de la Sección de Política Cultural del INLE, una Oficina para el Visado de Traducciones en la que colaboraban activamente los miembros del recién creado Cuerpo de Inspectores Técnicos de Traducción. De esta manera, la publicación de versiones españolas de obras extranjeras no solo requería la aprobación de su contenido y valor moral por parte de la Sección de Censura de Libros, 
sino también la valoración positiva de su calidad y pureza lingüísticas por parte de la Oficina para el Visado de Traducciones ${ }^{2}$.

En el presente artículo, se propone una primera aproximación a ese nuevo mecanismo que aseguraría la ilusión de una autarquía lingüística por la que se erradicaría toda influencia extranjera sobre el idioma español ${ }^{3}$.

\section{Confusión entre censura y visado}

El principal escollo que ha debido afrontar esta investigación ha sido la escasa bibliografía que, sobre este tema, se ha escrito tanto en el campo de la historia de la edición como en el campo de la historia y la crítica literarias. De hecho, resulta sorprendente constatar que la existencia del visado de traducciones no aparezca mencionada ni en el libro de Manuel L. Abellán, Censura y creación literaria en España (1939-1976), ni en la Historia de la Traducción en España (Lafarga y Pegenaute 2004), ni en los estudios sobre traducción y censura desarrollados desde la universidad española (Santoyo Mediavilla 1985; Rabadán 2000; Merino Álvarez 2007; Zaragoza Ninet, Martínez Sierra y Ávila-Cabrera 2015). Dichos estudios abordan el impacto de la censura sobre la práctica de la traducción pero desconocen la existencia de la Oficina para el Visado. Es más, en el ámbito del estudio de la literatura, se confunden sistemáticamente censura e inspección lingüística.

En el Bosquejo de una política del libro, Gustavo Gili y Roig (1944: 111) muestra el visado de traducciones como trámite burocrático distinto a los correspondientes a la censura, el permiso de circulación o la licencia de exportación. Valeriano Bozal (1969: 86) insiste en esta misma diferencia al enumerar, en no más de quince líneas, los distintos instrumentos de intervención del régimen en la política del libro. Para este autor, la censura constituiría el instrumento más conocido pero habría otros, complementarios, de "notable efectividad", a saber, el Servicio de Inspectores Técnicos de Traducción, la distribución de papel y la concesión de divisas para el pago de derechos a autores extranjeros.

En una importante revisión bibliográfica sobre censura editorial franquista, Andrés de Blas (1999: 292) menciona tímidamente, y "a falta de mayor confirmación", la existencia del Servicio de Inspectores de Traducción. También en nota al pie,

2 La descripción de la distribución oficial de competencias entre la Sección de Censura de Libros y la Oficina para el Visado de Traducciones no ha de ocultar el hecho de que muchos censores incluían de manera habitual, en sus informes, propuestas de corrección o valoraciones sobre la calidad de las traducciones. Así, por ejemplo, en el expedientes Y-561, relativo a la traducción de Félix Díez Mateo de la obra Eugenio Beauharnais, hijastro de Napoleón de Adalberto (1942), editado por Espasa-Calpe, el censor E. Frax indica que "bien por el texto o bien por la traducción existen algunos párrafos confusos y algunos errores no muy importantes" [AGA (3)50 21/06699]. Del mismo modo, en el expediente 6-649, correspondiente a la versión que Käthe von Blankestein y Enrique de Juan hicieron de la Crónica del callejón de los gorriones de Wilhelm Raabe (1943), el censor Conde determina que se trata de una "buena traducción" [AGA (3)50 21/07031].

Como se ha hecho en esta nota, se indicarán siempre entre corchetes las referencias a material de archivo. Cuando la referencia pueda resultar insuficiente, se completará la nota a pie de página con la información que permita identificar el documento.

3 Aunque, por su conocido lema de "Limpia, brilla y da esplendor", cabría esperar una participación activa de la Real Academia Española en esta tarea concreta de "purificación del idioma” (Montañés, 1947: 9) en las traducciones de los años 40, no hemos encontrado indicios de tal participación ni en las historias que de esta institución han escrito Víctor García de la Concha (2014) y Alonso Zamora Vicente (2015) ni en el catálogo de la exposición La lengua y la palabra: trescientos años de la Real Academia Española, comisariada en 2013 por María del Carmen Iglesias. 
Mònica Baró Llambias (2005: 55) indica que este servicio "otorgaba los visados correspondientes a las solicitudes de edición de obras extranjeras”.

Se incluyen breves comentarios sobre el Servicio de Inspectores Técnicos de Traducción en el volumen Los señores del libro de Eduardo Ruiz Bautista (2005: 260), en la Historia de la edición en España (1939-1975) dirigida por Jesús Antonio Martínez Martín (2015: 104, 238-239), así como en la tesis doctoral de Ana María Rodrigo Echalecu (2016: 39-41 y 149). Según esta autora, tras el visado "subyacía el principio del autarquismo cultural que demonizaba las traducciones de libros extranjeros como posibles contaminantes de los principios ideológicos del [Régimen]" (Rodrigo Echalecu 2015: 104). Encontramos esta misma idea en La batalla del libro en el primer franquismo, de Gabriel Andrés (2012: 47): "la traducción en sí representaba, inevitablemente, un elemento infectante dentro del proyecto de construcción de un ideario nacionalista".

En la mayoría de estos títulos, el interés por el Servicio de Inspectores Técnicos es secundario. Los autores recurren a él como prueba de la voluntad institucional de controlar el exceso de traducciones en la producción editorial de los años 40. Profundizan en este tema, aunque no mencionan el visado de traducciones, Xavier Moret (2002: 64-68) en su Historia de la edición en España y Jacqueline Hurtley (1992) en el estudio que dedica a la labor realizada por Josep Janés, el editor que más traducciones publicó en esa época.

Al igual que los estudios sobre la edición durante el franquismo, los ensayos sobre crítica e historia literarias aportan muy pocos datos sobre el Cuerpo de Inspectores Técnicos de Traducción. Así, por ejemplo, Fanny Rubio (1973: 444) se limita a mencionar su implantación al describir el contexto cultural de la poesía de los primeros años de postguerra. A su vez, al hablar de traducción y censura, Lucía Montejo (2013: 37-38) obvia la existencia de este servicio mientras que Cintia Santana (2013: 24) retoma la explicación que diera Valeriano Bozal en Cuadernos para el diálogo.

Por otro lado, circulan de un texto a otro ideas erróneas relativas a las funciones del cuerpo de Inspectores de Traducción. Para muchos autores, este servicio "vigilaba la importación [o la entrada clandestina] de libros extranjeros y se encargaba de salvaguardar la moral y la ortodoxia católica que imponía la política franquista" (Ruiz Soriano 1997: 28, García García 2006: 21-22, Rodríguez Cacho 2009: 379, Payne 2014: 40, Badri 2015: 25). Según otros autores, el servicio tenía por objetivo "vigilar cuantas [traducciones] intentaban ver la luz y que por lo tanto deberían ser visadas" (Sánchez Dueñas y Porro Herrera 2015: 251). Hay también quien le concede la competencia de establecer el catálogo de autores publicables: "En 1942 se crea el Servicio de Inspectores de Traducción: solo se traducirá a escritores de segunda fila, en modo alguno peligrosos" (Carrero 1977: 92). Todas estas citas denotan prejuicios con respecto al número de traducciones que se publicaban en España y revelan también una gran confusión entre censura, control de planes editoriales e inspección de traducciones.

Dada la escasez de referencias bibliográficas, el presente trabajo se ha ido construyendo a partir de otras fuentes documentales. Han sido de gran utilidad las disposiciones publicadas en el Boletín Oficial del Estado y las circulares de la Delegación Nacional de Propaganda, el Instituto Nacional del Libro Español y la Delegación del INLE en Barcelona. Entre las publicaciones periódicas consultadas, destaca Bibliografía Hispánica, que fue una de las herramientas con las que el INLE pretendía "introducir en el dominio del libro la gran política española de la Falange” (1942a: 1). 
En el Archivo General de la Administración de Alcalá de Henares (en adelante, AGA), es posible consultar algunos de los partes que Vicente Díaz Poyatos, secretario General del INLE, remitía mensualmente a la Delegación Nacional de Propaganda para informar de la labor realizada por dicho instituto. En esos partes y, más concretamente, en el apartado reservado a la Sección de Política Cultural, se ofrecen datos relativos a obras recibidas, apercibimientos o recibos de inspectores. Se indica también que el INLE disponía de un archivo de obras visadas e informes. Pero esos informes, que son pieza fundamental para conocer en detalle la labor realizada por los inspectores de traducción, no están disponibles en el AGA.

Tras la desaparición del INLE en 1986, se decreta el cierre de su archivo y toda la documentación que se había generado pasa a ser custodiada por el Centro de Documentación del Libro, la Lectura y las Letras Españolas, dependiente de la Subdirección General de Promoción del Libro, la Lectura y las Letras Españolas. En febrero de 2005, se reabre el archivo y se procede a su registro y catalogación. Sin embargo, el Centro de Documentación no dispone de los informes de la Oficina para el Visado de Traducciones ya que distintos siniestros fueron mermando los fondos $\mathrm{y}$, del periodo que nos ocupa, solo quedan unas pocas cajas relativas a gestión de personal.

Por otro lado, el Arxiu Nacional de Catalunya (en adelante, ANC), ubicado en Sant Cugat del Vallès, cuenta con gran parte de la documentación tramitada por la delegación que el INLE tenía en Barcelona. Lamentablemente, tampoco se hallan disponibles en esta institución los informes correspondientes a las traducciones visadas.

Dado que, aún no hemos logrado encontrar ninguno de los expedientes, por el momento no nos es posible ofrecer un análisis de su contenido ni una descripción detallada de las prácticas concretas de los Inspectores Técnicos de Traducción. Valga la información que se ofrece en las siguientes páginas como primera aproximación al estudio de la Oficina para el Visado de Traducciones.

\section{Creación de la Oficina para el Visado de Traducciones}

Por oficio de fecha 3 de junio de 1942, la Delegación Nacional de Propaganda ordenó al INLE la organización, con carácter "urgentísimo e imprescindible", de una Oficina de Traductores que "vigil[ase] la totalidad de las versiones al castellano de obras extranjeras, para cortar radicalmente la lamentable aparición de una serie de traducciones absolutamente inadmisibles y lesivas para la dignidad de nuestra lengua"4. Quedaba así fijado el objetivo de un nuevo mecanismo de control editorial que venía a sumarse a los ya existentes. Si la censura vigilaba que los contenidos y las ideas se ajustaran al dogma, a la moral, a la Iglesia, al Régimen y a sus instituciones, la Oficina de Traductores habría de asegurar la "corrección y dignidad del idioma español" (INLE 1942e) cuya "pureza", a juicio del Régimen, estaba siendo tratada de "manera vejatoria" en las traducciones de principios de los años 40 (INLE 1942g).

Siguiendo las órdenes de la Delegación Nacional de Propaganda, el INLE creó una Oficina para el Visado de Traducciones en el seno de la Sección de Política Cultural que, en ese momento, dirigía el novelista Darío Fernández Flórez y, más tarde, dirigiría el poeta Adriano del Valle (INLE 1942e y 1943c). Bajo la batuta de estos

[AGA, (3) 49.1 21/00068 - Legajo 1942-C59-76]. 
dos escritores, el INLE formó un Cuerpo de Inspectores de Traducción que actuaban como "corrector[es] de estilos ajenos", expurgando "los desafueros" y enmendando "los errores lesivos para la gloria imperial de nuestra bella lengua" (INLE 1943a).

Aunque las fechas son inciertas, las circulares del INLE aparecidas en prensa permiten suponer que la Oficina para el Visado de Traducciones estuvo operativa, al menos, entre el 1 de julio de 1942 y el 1 de noviembre de 1946 (INLE 1942b y 1946). Confirma su fecha de inicio de actividades un documento que forma parte de los fondos del INLE depositados en el Centro de Documentación del Libro en Madrid. Se trata del nombramiento de Antonio Márquez Pan como "Oficial de la Oficina para el Visado de Traducciones [...] con antigüedad y efectos económicos de uno de julio del año [de 1942] en curso"s.

\section{Traducciones inspeccionadas y traducciones exentas}

La normativa que había de regir el visado de traducciones sufrió numerosos cambios durante su primer año de vida. Según las instrucciones que entraron en vigor el 1 de julio de 1942, los editores tenían la obligación ineludible de someter a visado todas las traducciones que se hicieran al español de cualquier título extranjero, independientemente de su temática (INLE 1942c). Para ello, tenían que remitir a la Sección de Política Cultural del INLE un ejemplar de la obra en su idioma original acompañado de una copia en castellano, indicando siempre el nombre de la persona responsable de la traducción. En caso de obtener la aprobación deseada, los editores adquirían el compromiso de incluir "en lugar bien visible" y siempre "antes del comienzo del texto", es decir, en las primeras páginas de todos los ejemplares publicados, la siguiente frase: "Versión castellana autorizada por la Sección de Política Cultural del Instituto Nacional del Libro Español" (INLE 1942b y 1942d).

A principios del mes de julio, la Delegación del INLE en Barcelona ya anunciaba que "se cursar[ían] nuevas órdenes rectificando y simplificando el procedimiento de revisión" . Y así fue; antes de que acabara el mes, dejó de ser obligatoria la entrega de la obra original y se dio al editor la posibilidad de enviar las galeradas o un ejemplar de la traducción. Además, quedó derogada la obligación de incluir el texto indicativo de la aprobación por parte del INLE7

Poco después, el 21 de agosto de 1942, la Delegación de Barcelona completó las instrucciones iniciales indicando que las versiones al español debían entregarse "sin

\footnotetext{
[CDL, Fondo INLE - Sección de Personal - Caja 1705]

Circular n. ${ }^{\circ} 31757$ de 6 de julio de 1942 [ANC 27, 295: 22].

Circular n. 31881 de 31 de julio de 1942 [ANC 27, 295: 22]. Supimos de la derogación de esta norma durante nuestra primera visita a los fondos del Arxiu Nacional de Catalunya en enero de 2018. Meses antes, entre septiembre y diciembre de 2017, con la esperanza de encontrar algún libro que contuviera la frase que creíamos obligatoria, consultamos 173 traducciones en la biblioteca de la Facultad de Filología de la Universidad Complutense de Madrid y otras 763 en la Biblioteca Nacional de España; todas ellas aparecidas en la primera mitad de la década de los 40. Ninguno de estos ejemplares incluía la frase en cuestión. Ateniéndonos a los resultados de nuestra infructuosa búsqueda, creemos poder afirmar que nunca se llegó a aplicar esta norma. Sin embargo, se diría que, por algún motivo que desconocemos, en fechas posteriores sí hubo casos, no sistemáticos, en los que se aclaraba al lector que el libro que tenía entre las manos era una "traducción autorizada" aunque no se indicara por quién ni dentro de qué marco normativo. El carácter no sistemático de esta práctica puede verse, por ejemplo, en las versiones al español de las novelas de Pierre Benoit (1950abc), Madre y Rival, El fin y La pecadora, publicadas alrededor de 1950 por el editor Mateu: solo en dos de ellas se especifica que cuentan con autorización. No obstante, entendemos que, para ser publicadas, las tres tuvieron que obtener previamente el beneplácito de la censura.
} 
carta alguna, sino acompañadas de una nota o declaración extendida por triplicado, tamaño holandesa, con arreglo a [un] modelo [...]" en el que parece obviarse la obligación de indicar el nombre del traductor ${ }^{8}$ :

Editorial presenta en (manuscrito, galerada, volumen, mecanografiada) para su revisión, la versión de la obra (autor y título en castellano) traducida del (idioma del original)

Barcelona, .....

(sello)

En febrero de 1943, la Delegación de Barcelona volvía a ponerse en contacto con los editores catalanes para comunicarles que la obligación de solicitar el visado de traducción se extendía a "las reducciones y adaptaciones de las obras extranjeras que [hubieran] de publicarse" . Para evitar perjuicios económicos, quedaban exentas de visado las traducciones al castellano que estuvieran "en trámite de composición de impresión” en fecha anterior al 1 de julio de 1942 (INLE 1942d). Esta fecha límite fue pospuesta, primero, al mes de octubre ${ }^{10}$ y, luego, al 15 de noviembre (INLE 1942g).

Por otro lado, a partir del 28 de julio de 1942, se delegó en los editores "la responsabilidad del cuidado técnico y gramatical" de las versiones de obras científicas o técnicas redactadas originalmente en idioma distinto al castellano. A pesar de no necesitar visado, la calidad de estas traducciones seguiría siendo vigilada por la Sección de Política Cultural que tendría en cuenta "aquellas faltas de escrupulosidad para con la dignidad de nuestra lengua". El visado de traducciones quedaba así reservado para "obras literarias, filosóficas, históricas, religiosas y sobre temas de arte, etc.” (INLE 1942f).

\section{Tareas desarrolladas por la Oficina para el Visado}

A finales de marzo de 1943, la Delegación Nacional de Propaganda ordenó al INLE remitir un parte mensual de las actividades que hubiera desarrollado a partir del 1 de enero de ese mismo año ${ }^{11}$. La mayoría de estos partes se han perdido y solo unos pocos se conservan en el Archivo General de la Administración en Alcalá de Henares $^{12}$. Los partes incluyen un anexo con la labor efectuada desde la Delegación de Barcelona y su estructura refleja la división interna del INLE en diferentes secciones.

Dentro del epígrafe correspondiente a la Sección de Política Cultural, se reserva un apartado al "Visado de Traducciones". La información que allí se ofrece de manera esquemática es de carácter cuantitativo. Por este motivo, y dado que no siempre aparecen los mismos ítems ni siguen un patrón fijo, como si su desglose dependiera

\footnotetext{
Circular n. ${ }^{\circ} 32002$ de 21 de agosto de 1942 [ANC 27, 295: 22].

Circular n. ${ }^{\circ} 33515$ de 16 de febrero de 1943 [ANC 27, 294: 22].

Circular n. 31757 de 6 de julio de 1942 [ANC 27, 295: 22].

Oficio n. ${ }^{\circ} 1755$ de 8 de abril de 1943 [AGA (3) 49.1 21/00783].

En el Archivo General de la Administración (AGA) se conservan los partes correspondientes a los meses de enero, febrero, abril, mayo, junio, septiembre, octubre, noviembre y diciembre de 1943; y enero, marzo, abril, mayo, junio, agosto, septiembre, octubre, noviembre y diciembre de 1944 [AGA (3)49.1 21/00783; (3) 49.1 21/01312; (3)49.1 21/2356; (3)49.1 21/2358]. Faltan, por tanto, los correspondientes a los meses de marzo, julio y agosto de 1943, febrero y julio de 1944 y todos los de 1945 y 1946.
} 
del criterio personal de diferentes individuos, resulta difícil saber a qué se refiere cada uno de los ítems del listado. De manera global, la información ofrecida podría clasificarse de la siguiente manera:

- OBRAS VISADAS: los partes dan cuenta del número de traducciones recibidas, aprobadas, denegadas, suspendidas y pendientes de aprobación.

- Documentos y ESCRITOS DESPACHADOS: se trata de acuses de recibo de obras, anotaciones, autorizaciones de obras, avisos de envío de giros, avisos de envío de paquetes de obras, avisos de envío de obras para visar, devolución de páginas de obras visadas, envío de duplicados, informaciones, informes, oficios, oficios de apercibimiento, oficios de envío de obras visadas, oficios de envío de recibos de obras, oficios de informes, recibos de obras, reclamaciones de pago de derechos de visado, relaciones de obras resueltas y otros documentos varios.

- Ficheros: los partes hacen alusión a dos ficheros, uno de obras suspendidas y otro de obras visadas e informes.

- VARIOS: se incluyen en este apartado los recibos de inspectores, las circulares a libreros y a editores así como la relación de obras recibidas durante el año.

Tal y como puede verse en las tablas que se incluyen más adelante, durante sus primeros seis meses de funcionamiento, en la Oficina de Visado se inspeccionaron 223 traducciones (INLE 1943a) ${ }^{13}$. En 1943, este número ascendería a más de 700 obras y, en 1944, a más de 560. En cuanto al ritmo de trabajo, de media, en 1943 ingresaron cada mes algo más de 78 traducciones frente a las 56 obras recibidas mensualmente en 1944. La mayoría llegaban directamente al INLE y menos del $20 \%$ eran remitidas desde la Delegación de Barcelona.

Casi todas las traducciones presentadas obtuvieron aprobación (imaginamos que algunas con correcciones). Según los partes mensuales que manejamos, habrían sido rechazadas tan solo 21 traducciones en 1943 y 7 en 1944, lo que representa, respectivamente, el 2,9\% y el 1,2\% del total anual de traducciones inspeccionadas. A mediados de la década, los editores justificarán su solicitud de supresión del visado aludiendo precisamente al carácter residual del porcentaje de rechazos: "La mejor garantía para la corrección y pureza de las versiones españolas [es] la conciencia profesional de los mismos editores, bien patente en la mayoría de los casos, por encima de las excepciones censurables" (INLE 1946).

Tabla 1. Obras ingresadas, obras aprobadas, obras denegadas y apercibimientos (Elaborado tras revisión de los partes mensuales disponibles en el AGA)

\begin{tabular}{|c|c|c|c|c|c|c|c|c|c|c|c|c|c|}
\hline Año 1942 & 怘 & 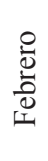 & 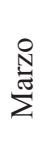 & $\begin{array}{l}\bar{E} \\
\end{array}$ & 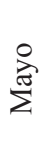 & $\stackrel{\stackrel{\varrho}{\Xi}}{\Xi}$ & $\stackrel{?}{\Xi}$ & $\begin{array}{l}\stackrel{0}{0} \\
\stackrel{0}{0} \\
80 \\
\&\end{array}$ & 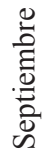 & 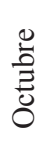 & 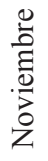 & 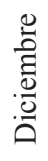 & 吾 \\
\hline Obras ingresadas & & & & & & & $\ldots$ & $\ldots$ & $\ldots$ & $\ldots$ & $\ldots$ & $\ldots$ & 223 \\
\hline
\end{tabular}

13 Este dato aparece recogido en el número de febrero de 1943 de la revista Bibliografía Hispánica: "Desde el día 1 de julio al 31 de diciembre pasado, nuestras estadísticas de obras visadas y minuciosamente expurgadas de errores idiomáticos, con lo que dábamos cumplimiento al espíritu y a la letra del oficio por el cual se creó el Cuerpo de Inspectores de Traducción, arrojan un total de 223 traducciones inspeccionadas" (INLE 1943a). 


\begin{tabular}{|c|c|c|c|c|c|c|c|c|c|c|c|c|c|}
\hline Año 1943 & 莺 & $\begin{array}{l}0 \\
\stackrel{0}{0} \\
\stackrel{0}{0}\end{array}$ & 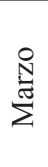 & $\begin{array}{l}\bar{E} \\
\bar{Z}\end{array}$ & 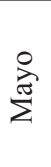 & 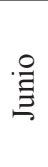 & $\stackrel{\varrho}{\Xi}$ & $\begin{array}{l}0 \\
\text { 劳 } \\
\text { 80 } \\
<\end{array}$ & 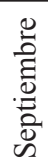 & $\begin{array}{l}0 \\
\frac{0}{E} \\
0 \\
0\end{array}$ & 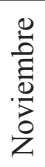 & 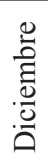 & $\stackrel{\bar{\Xi}}{0}$ \\
\hline Obras ingresadas & 56 & 135 & $\ldots$ & 84 & 78 & 64 & $\ldots$ & $\ldots$ & 79 & 63 & 73 & 74 & 706 \\
\hline Obras aprobadas & 54 & 132 & $\ldots$ & 53 & 48 & 55 & $\ldots$ & $\ldots$ & 52 & 56 & 51 & 69 & 570 \\
\hline Obras denegadas & 2 & 3 & $\ldots$ & 3 & 0 & 3 & $\ldots$ & $\ldots$ & 2 & 1 & 2 & 5 & 21 \\
\hline Apercibimientos & 15 & 8 & $\ldots$ & 0 & 0 & 0 & $\ldots$ & $\ldots$ & 0 & 0 & 4 & 10 & 37 \\
\hline
\end{tabular}

\begin{tabular}{|c|c|c|c|c|c|c|c|c|c|c|c|c|c|}
\hline Año 1944 & 喝 & 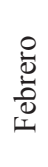 & $\begin{array}{l}\text { N } \\
\text { 荧 }\end{array}$ & $\bar{E}$ & 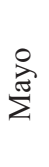 & 吕 & $\stackrel{\varrho}{\Xi}$ & 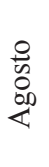 & 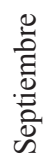 & $\begin{array}{l}0 \\
\stackrel{0}{0} \\
\text { D. } \\
0\end{array}$ & $\begin{array}{l}0 \\
0 \\
\text { है } \\
0 \\
0 \\
z\end{array}$ & 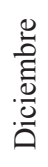 & 苞 \\
\hline Obras ingresadas & 54 & $\ldots$ & 96 & 48 & 49 & 53 & $\ldots$ & 61 & 47 & 68 & 51 & 39 & 566 \\
\hline Obras aprobadas & 38 & $\ldots$ & 90 & 47 & 47 & 41 & $\ldots$ & 61 & 46 & 64 & 50 & 39 & 523 \\
\hline Obras denegadas & 0 & $\ldots$ & 2 & 0 & 1 & 0 & $\ldots$ & 0 & 1 & 2 & 1 & 0 & 7 \\
\hline Apercibimientos & 2 & $\ldots$ & 0 & 0 & 0 & 0 & $\ldots$ & 0 & 0 & 0 & 1 & 0 & 3 \\
\hline
\end{tabular}

\begin{tabular}{|c|c|c|c|c|c|c|c|c|c|c|c|c|c|}
\hline $\begin{array}{l}\text { Obras remitidas desde } \\
\text { la Delegación del } \\
\text { INLE en Barcelona }\end{array}$ & 咅 & 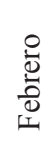 & 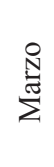 & $\begin{array}{l}\bar{E} \\
\bar{Z}\end{array}$ & 胥 & $\stackrel{̊}{\Xi}$ & $\stackrel{\varrho}{\Xi}$ & 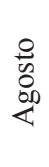 & 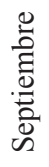 & 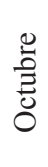 & 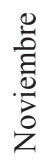 & 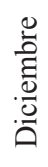 & $\underset{0}{\tilde{0}}$ \\
\hline 1943 & 15 & 25 & $\ldots$ & 12 & 9 & 14 & $\ldots$ & $\ldots$ & 6 & 11 & 14 & 7 & $113^{14}$ \\
\hline 1944 & 12 & $\ldots$ & 22 & 17 & 17 & 12 & $\ldots$ & 6 & 16 & 15 & $\ldots$ & $\ldots$ & 117 \\
\hline
\end{tabular}

\section{Tasas y sanciones}

Dado que el INLE no contaba con presupuesto específico para afrontar los gastos derivados de la contratación de los Inspectores Técnicos de Traducción, a partir del 1 de noviembre de 1942 las editoriales tuvieron que empezar a abonar, "contra recibo en la Administración del INLE", una tasa en concepto de derechos de visado de traducción por cada una de las obras que requirieran ser inspeccionadas (INLE 1942g). Un año más tarde, el 1 de octubre de 1943, el importe de esa tasa pasó de las 50 pesetas iniciales a 100 pesetas (INLE 1943b).

La norma preveía igualmente sanciones económicas tanto para aquellos editores que enviaban a imprenta una traducción que no había obtenido el correspondiente visado, como para aquellos impresores que aceptaban este tipo de encargos no autorizados (INLE 1942d). Según la gravedad del caso, la infracción podía ser castigada con un simple apercibimiento, una multa de entre 500 y 5000 pesetas o, incluso, la "recogida" [sic] de la edición (INLE 1942g).

14 En el Arxiu Nacional de Catalunya se conserva un informe de fecha 11 de diciembre de 1943 sobre los trabajos realizados durante ese mismo año por la Delegación de Barcelona [ANC 27, 138: 9]. Se indica en dicho informe que fueron 128 los "originales de traducciones cursadas para su visado". 
En los partes mensuales de actividades del INLE, figura la cantidad de oficios de apercibimiento emitidos. En enero de 1943, que parece ser el mes con el mayor número de infracciones, fueron amonestadas "por no haber pasado alguna de sus publicaciones por el negociado" las editoriales que se relacionan a continuación: Ameller, Blass, Bruguera, Cisne, Destino, Espasa-Calpe, Iberia, Lábor, Litúrgica Española, Maravilla, Marisal, Mediterráneas, Molino, Sopena y Tartessos ${ }^{15}$.

Según los datos de los que disponemos, el número de amonestaciones habría ido disminuyendo durante los dos primeros años de vida de la Oficina de Visado, registrándose ocho amonestaciones en febrero de 1943, diez en diciembre de 1943, dos en enero de 1944, y tan solo una en noviembre de 1944. Esta tendencia a la baja cambiaría un año después como prueba la circular de 3 de diciembre de 1945 en la que la Delegación del INLE en Barcelona comunica haber comprobado que "algunos editores han dejado de remitir para el visado de traducciones las obras publicadas en estos últimos tiempos"16.

\section{Los inspectores}

Poco sabemos de los inspectores técnicos para el visado de traducciones. En un primer momento, el INLE los describe desde un punto de vista académico resaltando su "autoridad intelectual y [su] absoluto dominio de nuestro idioma y de aquellas otras lenguas en que est[á]n escritas las obras que les son encomendadas" (INLE 1942g). Más tarde pone de manifiesto su condición de creadores: se trataría de "escritores de reconocida solvencia que, al par, pudiesen prestar sus actividades a la servidumbre de corrector de estilos ajenos" (INLE 1943a). En este sentido, los "recibos de inspectores", cuya tramitación reflejan algunos de los partes mensuales de la actividad realizada por la Sección de Política Cultural, podrían confirmar que la inspección de traducciones no era la actividad principal de estos profesionales ${ }^{17}$. También es de suponer que, al igual que sucedía con los censores, se les exigiera la máxima confidencialidad y discreción. No en vano, la suya pretendía ser una "misión eficaz, humilde y callada", una "labor anónima, perseverante y modesta" (INLE 1943a).

A pesar del anonimato y de los silencios, creemos haber identificado a uno de los inspectores en la figura de Eduardo Luis del Palacio Fontán (1943: 19), quien anunció su "nuevo oficio de censor de traductores del francés" en la apología que de estos hizo en la revista Bibliografía Hispánica en febrero de 1943. Es cierto que, en esta cita, el autor se refiere a sí mismo como censor y no como inspector, pero no tenemos constancia de que existieran censores nombrados específicamente para verificar

\footnotetext{
Parte mensual de los trabajos realizados por el INLE durante enero de 1943 [AGA (3) 49.1 21/00783].

Circular n. ${ }^{\circ} 40091$ de 3 de diciembre de 1945 [ANC 27, 294: 22].

Para conocer la relación nominal de inspectores técnicos, sus condiciones de trabajo así como la naturaleza de su vinculación laboral con el INLE, hemos intentado sin éxito acceder a una "relación de todo el personal de es[e] instituto, con expresión de la categoría, sueldos y emolumentos de que disfruta, así como de su antigüedad en el servicio". El 10 de septiembre de 1946, el Secretario General del INLE remitió al Secretario General de Propaganda un documento de estas características. En el Archivo General de la Administración se conserva el oficio de envío pero no la relación de personal [(3)49.1. 21/02358]. Por otro lado, los libros de entradas y salidas de la Delegación de Barcelona que hemos podido consultar no recogen ninguna referencia a los inspectores técnicos [ANC 27, 91: 101 y ANC 27, 91:107].
} 
la calidad de traducciones de títulos extranjeros y, además, sus palabras no aluden al control de la ideología y los contenidos de una obra sino a la revisión de la labor desarrollada por aquellos que se dedican al "difícil arte de traducir". En cualquier caso, la descripción de los inspectores técnicos que transcribíamos más arriba corresponde al perfil de este poeta, dramaturgo, profesor de la Escuela Oficial de Idiomas y catedrático de francés del Instituto Cardenal Cisneros de Madrid (ABC 1969: 47) ${ }^{18}$.

\section{Las quejas de los editores}

La aparición de un nuevo trámite para la publicación de una traducción debió alterar los ritmos y plazos de trabajo que se manejaban en las editoriales. Así queda reflejado en una carta de fecha 14 de julio de 1943, que dirigió el poeta Marià Manent a la editorial Juventud, interesándose, entre otros asuntos, por el visado de ciertas traducciones que él mismo había realizado ${ }^{19}$. En la carta, Manent, que desde 1942 era también director literario de la editorial (Moret 2002: 65), solicita que se agilice el envío de las pruebas a la "oficina de traducciones" para "no retardar su publicación":

Le agradeceré se ocupe de que, una vez mecanografiada la traducción de Just so Stories (en castellano: Precisamente asi) [de Kipling], que estaba revisando el Sr. Corral, se remita rápidamente a la oficina de traducciones, pues el libro está ya componiéndose y conviene no retardar su publicación. También conviene pedir a Clarassó doble prueba del San Francisco, de Chesterton, para someter la traducción a la referida oficina.

La Delegación del INLE en Barcelona intentaba tranquilizar a los editores catalanes indicando que "la revisión de la versión de las obras literarias se efectuar[ía] con toda rapidez" 20 y que se realizaría "un envío a Madrid cada fin de semana [con] todas las declaraciones y todos los originales [reunidos] durante el indicado lapso de tiempo"21. Sin embargo, en al menos una ocasión, el retraso derivado de la acumulación de trámites parece haber ido acompañado de una demora en la expedición del visado con el consiguiente perjuicio económico para la editorial. Tal fue la circunstancia que llevó a Manent a elevar una queja ante el Secretario de la Delegación Provincial del INLE en Barcelona en junio de $1943^{22}$. En su caso, el retraso en la recepción de las correcciones propuestas

18 Eduardo Luis del Palacio Fontán se casó con la pianista María Luisa Chevalier. Su hijo, Carlos del Palacio Chevalier, se casó, a su vez, con Elisa Solá Dadín, con la que tuvo tres hijas. Una de ellas, de nombre Purificación, contrajo matrimonio con el político Manuel Soto, quien con el tiempo se convertiría en alcalde de Vigo. Durante la conversación telefónica que mantuvimos con este último en febrero de 2018, se nos indicó que la familia no conserva los archivos del antiguo inspector de traducción.

19 Carta de Marià Manent a la Editorial Juventud con fecha de 14 de julio de 1943 [ANC 1-361: 206]. Según los documentos que se encuentran disponibles en los fondos que la editorial Juventud tiene depositados en el Arxiu Nacional de Catalunya, no parece que el visado de traducciones haya afectado a los contratos firmados entre editoriales y traductores. De hecho, el contrato que firmara en 1943 Manuel Vallavé por su traducción de Le meraviglie del Mare de Giuseppe Colosi coincide exactamente con el contrato que firmó Adolfo Jordá en 1928 por la traducción de Casada por piedad de Hedwig Courths-Mahler [ANC 1-361]. Coinciden también los recibís firmados por Mercedes Audouard en 1930 y Agustín de Esclasans en 1945 [ANC 1-361].

20 Circular n. ${ }^{\circ} 31881$ de 31 de julio de 1942 [ANC 27, 295: 22].

21 Circular n. 32002 de 21 de agosto de 1942 [ANC 27, 295: 22].

22 Esta carta de 9 de junio es el único documento que hemos encontrado en el que se indica claramente la existencia de una labor correctora por parte de los inspectores de traducción [ANC 1-361: 205]. 
por la Oficina de Visado conllevó que la editorial Juventud lanzara al mercado una traducción de los Documentos póstumos del Club Pickwick (Dickens 1943c) varios meses después de que lo hicieran otras dos editoriales ${ }^{23}$ :

Nos permitimos exponerle un asunto relacionado con el Departamento de Traducciones, cuya urgente resolución le estimaremos tenga la bondad de encarecer, ya que las dilaciones producidas han ocasionado a esta Editorial considerables perjuicios.

En comunicación de dicho Departamento de Traducciones, de fecha 6 de febrero ppdo., nos anunciaba la devolución de las pruebas correspondientes a las obras Documentos póstumos del Club Pickwick, de Carlos Dickens, y Stein, de Constantino de Grünwald, en cuyas pruebas dicho Departamento había señalado algunas correcciones. En vista de que no llegaban las pruebas repetidamente aludidas, insistimos en la solicitud de las mismas, ya que sin conocer las correcciones señaladas por el Departamento de Traducciones, no podíamos activar la impresión de las citadas obras. Pero han transcurrido cuatro meses sin que llegaran a nuestro poder las pruebas en cuestión y entretanto se han puesto a la venta dos ediciones del libro de Dickens, lo que representa un grave obstáculo para el éxito de nuestra edición del Pickwick y se traduce en innegable quebranto económico.

En vista de que nuestras reiteradas solicitudes no surtían efecto alguno, con fecha 2 del actual hemos remitido al Sr. Presidente del I.N.L.E. nuevas pruebas de las obras mencionadas, rogándole se indiquen otra vez en las mismas las correcciones que se deben efectuar. Agradeceremos a Vd. vivamente tenga la bondad de gestionar la rápida solución de este asunto, por lo que le anticipamos las más expresivas gracias.

Los partes mensuales del INLE recogen el número de obras que, recibidas durante cada mes, permanecieron "pendientes [de visado]". Sin embargo, no permiten determinar la cantidad de solicitudes que sufrieron una demora tan importante como la que generó la queja de Marià Manent ya que no se identifica el momento en que fueron finalmente aprobadas o denegadas. En cualquier caso, el porcentaje de retrasos, que variaba de mes a mes, llegó a alcanzar, en abril y mayo de 1943, el 23,5 \% de las obras registradas.

\section{Tabla 2. Porcentaje de obras pendientes de visado (Elaborado tras revisión de los partes mensuales disponibles en el AGA)}

\begin{tabular}{|c|c|c|c|c|c|c|c|c|c|c|c|c|}
\hline Año 1943 & 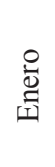 & 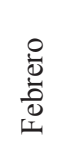 & 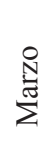 & $\overline{\overrightarrow{0}}$ & 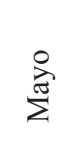 & 怘 & $\stackrel{\varrho}{\Xi}$ & 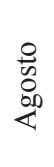 & 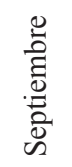 & $\begin{array}{l}0 \\
\text { Ẽ } \\
\text { O }\end{array}$ & 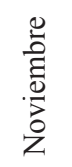 & 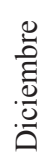 \\
\hline Obras ingresadas & 56 & 135 & $\ldots$ & 84 & 78 & 64 & $\ldots$ & $\ldots$ & 79 & 63 & 73 & 74 \\
\hline Obras pendientes & 0 & 0 & $\ldots$ & 28 & 30 & 6 & $\ldots$ & $\ldots$ & 25 & 6 & 20 & 0 \\
\hline Porcentaje (\%) & 0 & 0 & $\ldots$ & 23,5 & 23,4 & 3,8 & $\ldots$ & $\ldots$ & 19,7 & 3,78 & 14,6 & 0 \\
\hline
\end{tabular}

23 Al final, en 1943, fueron cuatro las editoriales que publicaron una versión al español de The Posthumous Papers of the Pickwick Club: Espasa-Calpe (Dickens 1943a), Tartessos (Dickens 1943b), Juventud (Dickens 1943c) y Lauro (Dickens 1943d). Desconocemos el momento exacto en que apareció cada una. 


\begin{tabular}{|c|c|c|c|c|c|c|c|c|c|c|c|c|}
\hline Año 1944 & 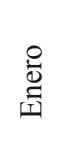 & 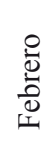 & $\stackrel{\stackrel{N}{\mathrm{~J}}}{\stackrel{\Xi}{\Sigma}}$ & $\overline{\bar{z}}$ & 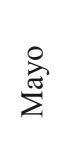 & ·号 & $\stackrel{\ominus}{\Xi}$ & 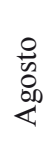 & 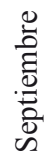 & $\begin{array}{l}0 \\
\text { : } \\
\text { Dू }\end{array}$ & $\begin{array}{l}0 \\
0 \\
\text { है } \\
.0 \\
\text { z } \\
\text { Z }\end{array}$ & 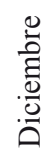 \\
\hline Obras ingresadas & 54 & $\ldots$ & 96 & 48 & 49 & 53 & $\ldots$ & 61 & 47 & 68 & 51 & 39 \\
\hline Obras pendientes & 16 & $\ldots$ & 9 & 1 & 1 & 12 & $\ldots$ & 0 & 0 & 2 & 0 & 0 \\
\hline Porcentaje (\%) & 8,6 & $\ldots$ & 8,6 & 0,4 & 0,4 & 6,3 & $\ldots$ & 0 & 0 & 1,3 & 0 & 0 \\
\hline
\end{tabular}

Como puede verse en la tabla, el porcentaje de obras pendientes de visado disminuyó considerablemente a lo largo de 1944. Ese mismo año, la Delegación Nacional de Propaganda decidió simplificar los trámites de censura para las traducciones que debían lanzarse en la Feria Nacional del Libro. Si en ese momento no se modificó el servicio del Visado de Traducciones fue porque, a juicio del INLE, "la rapidez de sus trámites no entra[ba] en pugna con los que se [querían] simplificar con [esas] disposiciones" (INLE, 1944). Por su parte, los editores, que no compartían esa impresión, abogaban abiertamente por la desaparición de los "engorrosos trámites burocráticos [y las] reglamentaciones sobreabundantes e innecesarias" que precedían la publicación de un libro (Gili y Roig 1944: 111).

\section{Fin del visado y disolución del cuerpo de inspectores}

Atendiendo a los ruegos de los editores, en marzo de 1944, se decidió suprimir el trámite previo de censura para determinadas obras, entre ellas las de carácter técnico y científico ${ }^{24}$. Además, dado que el papel había vuelto a ser declarado como de venta libre, se aprobó, en el mes de septiembre, la supresión del servicio de presentación de los planes semestrales de edición ${ }^{25}$. El visado de traducciones tardaría aún dos años más en dejar de ser obligatorio para obras no exentas.

A partir de noviembre de 1946, el visado quedaba a cargo de toda editorial que comunicase por escrito al INLE su deseo de garantizar, bajo su propia responsabilidad, la "corrección y pureza" de las traducciones. De esta manera, la Sección de Política Cultural limitaba su labor de inspección a las obras de los editores que no hubieran realizado la comunicación anterior o que hubieran perdido su facultad de visado por incapacidad o desinterés (INLE 1946) ${ }^{26}$.

A juzgar por el esquema de organización del Instituto Nacional del Libro Español incluido en la Memoria de Actividades de la Dirección General de Propaganda publicada en 1947, la eliminación de la obligatoriedad del visado de traducciones habría conllevado la desaparición de la Oficina de Visado y la disolución del Cuerpo de Inspectores Técnicos. No tenemos pruebas de ello pero solo cuatro actividades dependían entonces de la Sección de Política Cultural: las exposiciones, la semana del libro, el censo de autores y el concurso de los 50 libros mejor impresos del año.

24 Disposición de 25 de marzo de 1944 de la Vicesecretaría de Educación Popular estableciendo el régimen de censura de las publicaciones que se indican, Boletín Oficial del Estado, 98, de 7 de abril de 1944, 2786.

25 Orden de 21 de septiembre de 1944 relacionada con el Instituto Nacional del Libro Español, Boletín Oficial del Estado, 296, de 22 de octubre de 1944, 7943.

26 Véase también la circular n. ${ }^{\circ} 42846$ de 26 de noviembre de 1946 [ANC 27, 294: 22]. 
Para algunos investigadores, la función específica de control de la calidad de las traducciones habría pasado al Servicio de Inspección de Libros encargado de la censura editorial (Andrés de Blas 1999: 295 n. 34; Berger 2011: 16). Sin embargo, hay varios documentos posteriores a la reorganización que sufrió el INLE en el año 1957 que apuntan en sentido contrario. Así, por ejemplo, Domingo Manfredi Cano indica que al nuevo Departamento de Asuntos Editoriales correspondía, entre otros, "lo relativo al régimen de propiedad intelectual, derechos de autor y traducción, depósito legal, represión del fraude editorial, control de las traducciones, ediciones musicales, grabaciones, etc." (Manfredi Cano 1963: 9). De igual modo, según el acta de la reunión celebrada el 18 de noviembre de 1957 por el Consejo de Administración del INLE, un representante de la Asociación de Traductores debía formar parte de la Comisión Delegada para Asuntos Editoriales [AGA (3) 49.1. 21/6046].

\section{Conclusiones}

Como hemos expuesto, entre 1942 y 1946, funcionó en España la Oficina para el Visado de Traducciones. A través de esta, el Régimen franquista pretendió garantizar la pureza de la lengua utilizada en las traducciones de obras extranjeras, erradicando de ellas toda posible incorrección o influencia.

El visado es, sin duda, menos conocido que otros mecanismos de control del libro como la censura, la distribución de papel, la revisión de planes editoriales o la concesión de divisas para el pago de derechos de autor. Sin embargo, no debería obviarse la existencia de la Oficina ni confundirse sus funciones con las que tenía atribuidas la Sección de Censura de Libros.

A pesar de la escasa documentación que, sobre este asunto, se conserva en los diferentes archivos, el estudio de la Oficina para el Visado de Traducciones y el Cuerpo de Inspectores Técnicos de Traducción resulta de gran relevancia para los estudios de traducción, la literatura comparada y la historia del libro, pues aporta nuevos elementos para una mayor comprensión de la intervención del Régimen sobre la edición y la configuración del campo literario español.

\section{Archivos y centros de documentación consultados}

AGA = Archivo General de la Administración, Alcalá de Henares.

$\mathrm{ANC}=$ Arxiu Nacional de Catalunya, Fondos Editorial Juventud y Delegación del

INLE en Barcelona, Sant Cugat del Vallès.

$\mathrm{BNE}=$ Biblioteca Nacional, Madrid.

$\mathrm{CDL}=$ Centro de Documentación del Libro, Fondo INLE, Madrid.

\section{Referencias}

$A B C$, "Sobre el crecimiento de la producción editorial", Madrid, 3 de junio (1944), 10. $A B C$, "Don Eduardo del Palacio", Madrid, 1 de mayo (1969), 46-47.

Abellán, M. L., Censura y creación literaria en España (1939-1976). Barcelona:

Edicions 621980. 
Adalberto, Príncipe de Baviera, Eugenio Beauharnais, hijastro de Napoleón, trad. de Félix Díez Mateo. Madrid: Espasa-Calpe 1942.

Andrés, G., La batalla del libro en el primer franquismo. Madrid: Huerga y Fierro 2012.

Andrés de Blas, J., "El libro y la censura durante el franquismo: un estado de la cuestión y otras consideraciones", Espacio, Tiempo y Forma, Historia Contemporánea, V.12 (1999), 281-301.

Badri Guezza, N., Aspectos arábigo-andaluces en la producción literaria de Antonio Gala, Tesis doctoral. Madrid: UCM 2015. http://eprints.ucm.es/39728/.

Baró Llambias, M., Les edicions infantils i juvenils de l'éditorial Joventut (19231969), Tesis doctoral. Barcelona: Universidad de Barcelona 2005. http://diposit. ub.edu/dspace/handle/2445/35852.

Benoit, P., El fin, trad. Montserrat Rifá de Molas. Barcelona: Mateu c. 1950a.

Benoit, P., Madre y Rival, trad. R. Ballester Escalas. Barcelona: Mateu c. 1950b.

Benoit, P., La pecadora, trad. F. Ortega. Barcelona: Mateu c. 1950c.

Berger, L., Das Pressegesetz von 1966 in seiner Anwendung auf katalanische und kastilische Publikationen, Magistra der Philosophie. Viena: Universidad de Viena 2011. https://othes.univie.ac.at/14177/.

Bozal, V., "La edición en España. Notas para su historia”, Cuadernos para el diálogo 14 extraordinario (1969), 85-93.

Carrero Eras, P., "Notas para una sociología de la cultura literaria en España desde 1939”, Revista española de la opinión pública, 47 (1977), 91-121.

DGP = Dirección General de Propaganda, Memoria de las actividades de la Dirección General de Propaganda. Madrid: Publicaciones Españolas 1947.

Dickens, C., Papeles póstumos del Club Pickwick, trad. de Manuel Ortega y Gasset. Madrid: Espasa-Calpe 1943a.

Dickens, C., Los papeles póstumos del club Pickwick, trad. de María Héctor y Diego Navarro. Barcelona: Tartessos 1943b.

Dickens, C., Documentos póstumos del Club Pickwick, trad. de Juan de Paso. Barcelona: Juventud 1943c.

Dickens, C., Documentos póstumos del Club Pickwick, trad. de José Luis del Río. Barcelona: Lauro 1943d.

García de la Concha, V., La Real Academia Española. Vida e historia. Madrid: Espasa Calpe 2014.

García García, A. G., Antología de la poesía española (1939-1975). Madrid: Akal 2006.

Gili y Roig, G., Bosquejo de una política del libro. Barcelona: G.G. 1944.

Herrero, M., "Estadísticas y comentarios", Bibliografia Hispánica, 1, 5 (1942), 1-7.

Hurtley, J., Josep Janés, editor de literatura inglesa. Barcelona: PPU 1992.

Iglesias, M. C. (comisaria), La lengua y la palabra: trescientos años de la Real Academia Española. Madrid: Real Academia Española 2013.

INLE = Instituto Nacional del Libro Español, [Texto de presentación], Bibliografía Hispánica, 1.1 (1942a), 15-21.

INLE, "Nota del Instituto Nacional del Libro Español a los Editores", $A B C$, Sevilla, 6 de junio (1942b), 9.

INLE, "Normas sobre el visado de traducciones", $A B C$, Madrid, 14 de junio (1942c), 16.

INLE, "Aviso a todos los editores", $A B C$, Madrid, 10 de julio (1942d), 14.

INLE, "Reunión de la Junta de gobierno. Nota a los editores sobre la intervención de planes editoriales", $A B C$, Madrid, 31 de julio (1942e), 10. 
INLE, "Nota a los editores sobre el visado de traducciones de obras técnicas", $A B C$, Madrid, 31 de julio (1942f), 10.

INLE, "El visado de las traducciones", $A B C$, Madrid, 1 de noviembre (1942g), 18.

INLE, "Pago de traducciones", Bibliografía Hispánica 1.4 (1942h), 41.

INLE, "Visado de traducciones", Bibliografía Hispánica 2.2 (1943a), 32.

INLE, "Circular n. " 51", Bibliografia Hispánica 2.8 (1943b), 49.

INLE, “Circular n. " 57. Traducciones”, Bibliografia Hispánica 2.9 (1943c), 82-83.

INLE, "Circular n. o 70: Traducciones", Bibliografia Hispánica 3.4 (1944), 305.

INLE, "Visado de traducciones", Bibliografía Hispánica 5.11 (1946), 750.

Lafarga, F. y Pegenaute, L. (eds.), Historia de la traducción en España. Salamanca: Editorial Ambos Mundos 2004.

Manfredi Cano, D., Instituto Nacional del Libro Español. Madrid: Sucesores de Rivadeneyra 1963.

Martínez Martín, J. A. (dir.), Historia de la edición en España 1939-1975. Madrid: Marcial Pons 2015.

Merino Álvarez, R. (ed.), Traducción y censura en España (1939-1985). Estudios sobre corpus TRACE: cine, narrativa y teatro. Bilbao: Servicio Editorial de la Universidad del País Vasco 2007.

Montañés, L., "Punto final en lo de las traducciones", El Español. Semanario de la política y del espíritu, 15 de marzo (1947), 9.

Montejo Gurruchaga, L., Discurso de autora: género y censura en la narrativa española de posguerra. Madrid: UNED 2013.

Moret, X., Tiempo de editores. Historia de la edición en España, 1939-1975. Barcelona: Ediciones Destino 2002.

Palacio Fontán, E. L. del, "Grandeza y servidumbre del Traductor", Bibliografía Hispánica, 2.2 (1943), 19-30.

Payne, Z., La relación entre la filosofía y la poesía en la posguerra española: el caso de José Luis Hidalgo, Tesis Doctoral. Madrid: UCM 2014 http:/eprints. ucm.es/27529/.

Raabe, W., Crónica del callejón de los gorriones, trad. de Käthe von Blankestein y Enrique de Juan, Barcelona: Tartessos 1943.

Rabadán, R. (ed.), Traducción y censura inglés-español:1939-1985. Estudio preliminar. León: Universidad de León 2000.

Rodrigo Echalecu, A. M., "Los organismos del libro y el corporativismo editorial. El Instituto Nacional del Libro Español”, en: Martínez Martín, J.A. (dir), Historia de la edición en España 1939-1975. Madrid: Marcial Pons 2015, 97-119.

Rodrigo Echalecu, A. M., La politica del libro durante el primer franquismo, Tesis Doctoral. Madrid: UCM 2016. http://eprints.ucm.es/39125/.

Rodríguez Cacho, L., Manual de Historia de la Literatura española 2, Siglos XVIII al XX [hasta 1975]. Madrid: Castalia Universidad 2009.

Rubio, F., "La poesía española en el marco cultural de los primeros años de posguerra", Cuadernos Hispanoamericanos 276 (1973), 441-467.

Ruiz Bautista, E., Los señores del libro: propagandistas, censores y bibliotecarios en el primer franquismo. Gijón: Ediciones Trea 2005.

Ruiz Soriano, F., La poesía de postguerra. Vertientes poéticas de la primera promoción. Barcelona: Literatura y Ciencia 1997.

Sánchez Dueñas, B. y Porro Herrera, M. J., Concha Lagos, agente cultural: los “Cuadernos de Ágora”. Madrid: UNED 2015. 
Santana, C., Forth and back: translation, dirty realism and the Spanish novel (19751995). Lewisburg: Bucknell University Press 2013.

Santoyo Mediavilla, J. C., El delito de traducir. León: Universidad de León 1985.

Zamora Vicente, A., La Real Academia Española. Madrid: Real Academia Española-Fundación María Cristina Masaveu Peterson 2015.

Zaragoza Ninet, M. G., Martínez Sierra, J. J. y Ávila-Cabrera, J. J. (eds.), "Traducción y censura: nuevas perspectivas", Quaderns de Filologia: Estudis Literaris, XX (2015). https://dialnet.unirioja.es/ejemplar/411354 